\title{
A Case Report of Congenital High Airway Obstruction Syndrome (CHAOS) caused by Complete Laryngeal Obstruction.
}

\author{
Rahman $\mathrm{T}^{1}$, Khalequezzaman $\mathrm{S}^{2}$, Ahsan $\mathrm{S}^{3}$, Alam $\mathrm{J}^{4}$, Sarker $\mathrm{MK}^{5}$
}

\begin{abstract}
CHAOS or Congenital High Airway Obstruction Syndrome is a blockage of the fetus's trachea or larynx due to a number of factors including narrowing of the airway, a web-like membrane or even tracheal atresia. In the uterus, the fetal lungs constantly produce fluid and as a result of this airway blockage in the trachea, the lung fluid cannot escape out of the fetal mouth. Because of this the fetus's lungs become distended with fluid and over distended lungs can put pressure on the heart and affect the heart's ability to function. If the heart cannot beat effectively hydrops or congestive heart failure can occur. We present a case of CHAOS with hydrops and associated anomalies, determined prenatally at about 21 weeks by MRI in our hospital.
\end{abstract}

\section{Keywords}

Congenital high airway obstruction syndrome (CHAOS), laryngeal obstruction.

\section{Introduction}

Congenital high airway obstruction sequence (CHAOS) is a rare, life-threatening condition characterized by complete or near complete intrinsic obstruction of the fetal airway. CHAOS has been considered an almost invariably fatal condition. However, advances in prenatal imaging, earlier diagnosis in utero, in utero surgical treatment, and delivery via ex utero intrapartum treatment (EXIT) to tracheostomy may contribute to improving the outcome of this condition. We present a case of antenatally diagnosed CHAOS with associated anomalies and literature review.

\section{Case Report}

A 29-years women (gravida 2, para 0) was referred to the Apollo Hospitals Dhaka at 21+ weeks of gestation for evaluation of a fetal hydrops. Her menstruation had been on a regular cycle of 28-30 days. She had history of spontaneous abortion at 16 weeks, 3 years back. No consanguineous marriage between them and have good medical history. There is no history of babies born with congenital anomalies in her or her husband's family. Her ELISA for TORCH infection revealed no recent infection. She did not have any genetic study done for her recent and previous pregnancy.

Ultrasound examination was carried out outside AHD and was diagnosed as hydrops fetalis. The patient was evaluated with fetal MRI \& Doppler USG in our hospital. Fetal MRI was carried out on a 1.5 Tesla scanner (Siemens, Germany) using T2 weighted (HASTE) \& TrueFISP sequences in sagittal, Coronal and axial planes which showed large markedly hyperintense lungs (as compared to fetal skeletal muscle) causing inversion of diaphragm (Fig1, a \& b) and compression of heart (Fig 2). Dilated trachea containing fluid 

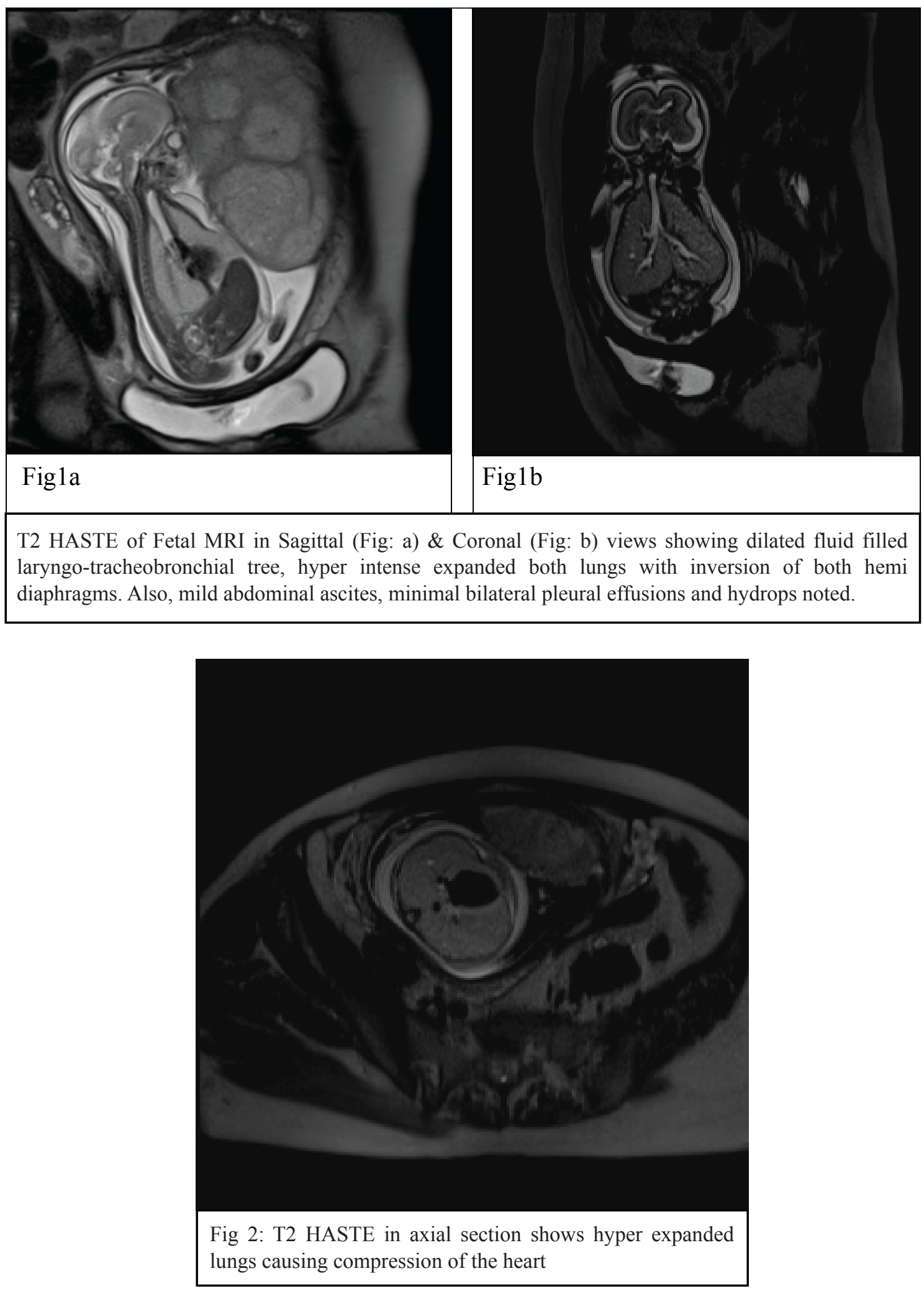
within with a gap at the level of larynx (subglottic region) with mild ascites and bilateral minimal pleural effusions (Fig1, a \& b). Associated findings were horseshoe kidneys and absent stomach fluid with oligohydramnios and also hydrops. These findings were strongly suggestive of CHAOS with fetal hydrops and associated with other congenital anomalies. The patient and her husband were counseled about the natural history and prognosis of CHAOS. They decided to terminate the pregnancy. The patient delivered vaginally a breech-presented stillborn female baby.

\section{Discussion}

Congenital high airway obstruction syndrome (CHAOS) is a very rare congenital malformation which takes place by deficient recanalization of the upper airways around the 10th week of gestation. ${ }^{1}$ In a healthy fetus, the fluid secreted by fetal lung is absorbed through the tracheobronchial tree. However, in case of any obstruction in the tracheobronchial tree, this fluid cannot be cleared. The accumulation of the fetal lung fluid results in gradual increase of intratracheal pressure leading to enlargement of the lungs. It is the beginning of a chain reaction: the enlarged lungs cause compression of the heart and great veins. Due to the compression, the heart replaces centrally and becomes small and dysfunctional. Decreased venous return and dysfunctional cardiovascular system end in ascites and hydrops. The diaphragm flattens or inverts according to the severity of the process. ${ }^{2}$ Besides tracheal atresia, the other rare underlying causes of
CHAOS are laryngeal agenesis, subglottic stenosis or atresia, and laryngeal webs or cysts. However, the obstruction is mostly secondary to laryngeal atresia. 1,3

The identification of airway obstruction is key to establishing the diagnosis of CHAOS and to differentiating it from bilateral lung masses such as a bilateral congenital pulmonary airway malformation (CPAM) or other causes of extrinsic airway obstruction such as a double aortic arch. ${ }^{4}$ Though sonography is the main diagnostic tool, MRI can help to better localize the level of obstruction. ${ }^{5,6}$ As a natural conclusion of the pathological process, bilateral large hyperechoic lungs, small, compressed, and centrally replaced heart, flattened or inverted diaphragm, and ascites are characteristic findings on sonographic examination. ${ }^{7}$ Regarding the amniotic fluid index, compression of the esophagus by dilated airways may lead to polyhydramnios, as the fetal swallowing of the fluid is disrupted. On the one hand, impaired swallowing of the fetus may also cause oligohydramnios. ${ }^{2}$ But the amniotic fluid quantity is not a constant marker for the diagnosis [B-REPORT]. The gestational age at the diagnosis may affect the amniotic fluid quantity. In this case, MRI showed an abnormally dilated airway distal to the level of obstruction. These findings are not easily identified by ultrasound.

Most cases of CHAOS are sporadic; however, several genetic syndromes have been linked with CHAOS. Moreover, the most commonly associated genetic syndrome is Fraser 
syndrome, which is characterized by a variable expression of laryngeal atresia, cryptophthalmos, syndactyly, renal agenesis, and abnormalities of the ears and external genitalia. ${ }^{8}$ Fetal MRI has also been an important tool in identifying these associated abnormalities and suspect genetic syndromes. The accurate identification of associated syndromes helps with management decisions and parental counseling.

The prenatal natural history and postnatal course of CHAOS often depends on whether the airway obstruction is complete. An ex utero intrapartum treatment (EXIT) procedure may offer potential for salvage. ${ }^{9}$ Overall prognosis is considered generally poor ${ }^{10}$, especially in Bangladesh where there is lack of expertise and equipment set up.

\section{Conclusion}

MRI shows a consistent pattern of abnormalities in fetus with CHAOS, accurately identifies the level of airway obstruction, and helps differentiate from other lung abnormalities such as bilateral congenital pulmonary airway malformation by demonstrating an abnormally dilated airway distal to the obstruction.

\section{References}

1 Roybal JL, Liechty KW, Hedrick HL. Predicting the severity of congenital high airway obstruction syndrome. Journal of Pediatric Surgery. 2010;45(8):1633-39.

2 Joshi P, Satija L, George RA. Congenital high airway obstruction Syndrome: antenatal diagnosis of a rare case of airway obstruction using multi modality imaging. Medical Journal Armed Forces India. 2012; 68(1):78-80. 3 Courtier J, Poder L, Wang ZJ, Westphalen AC, Yeh BM, Coakley FV. Fetal tracheolaryngeal airway obstruction: prenatal evaluation by sonography and MRI. Pediatric Radiology. 2010;40(11):1800-05.

4 Shum DJ, Clifton MS, Coakley FV, Hornberger LK, Joe BN, Goldstein RB, et al. Prenatal tracheal obstruction due to double aortic arch: a potential mimic of congenital high airway obstruction syndrome. AJR Am J Roentgenol. 2007;188:82-85.

5 Mong A, Johnson AM, Kramer SS. Congenital high airway obstruction syndrome: MR/US findings, effect on management, and outcome. Pediatr Radiol. 2008;38:1171-79.

6 Guimaraes CV, Linam LE, Kline-Fath BM. Prenatal MRI findings in congenital high airway sequence (CHAOS). Korean J Radiol. 2009;10:129-34.

7 Garg M. Case report: antenatal diagnosis of congenital high airway obstruction syndrome. Laryngeal atresia. Indian Journal of Radiology and Imaging. 2008;18(4):350-51.

8 Berg C, Geipel A, Germer U, Pertersen-Hansen A, Koch-Dorfler M, Gembruch U. Prenatal detection of Fraser syndrome without cryptophthalmos: case report and review of the literature. Ultrasound Obstet Gynecol. 2001;18:76-80.

9 Shimabukuro F, Sakumoto K, Masamoto H. A case of congenital high airway obstruction syndrome managed by ex utero intrapartum treatment: case report and review of the literature. Am J Perinatol. 2007;24(3):197-201.

10 Cavoretto P, Molina F, Poggi S. Prenatal diagnosis and outcome of echogenic fetal lung lesions. Ultrasound Obstet Gynecol. 2008;32(6):769- 83. 